\title{
Wireless Communications in Smart Rail Transportation Systems
}

\author{
César Briso-Rodríguez, ${ }^{1}$ Ke Guan, ${ }^{2,3}$ Yin Xuefeng, ${ }^{4}$ and Thomas Kürner ${ }^{3}$ \\ ${ }^{1}$ ETSIS Telecomunicacion, Technical University of Madrid, 28031 Madrid, Spain \\ ${ }^{2}$ State Key Laboratory of Rail Traffic Control and Safety, Beijing Jiaotong University and Beijing Engineering Research Center of \\ High-Speed Railway Broadband Mobile Communications, Beijing 100044, China \\ ${ }^{3}$ Institut für Nachrichtentechnik, Technische Universität Braunschweig, 38106 Braunschweig, Germany \\ ${ }^{4}$ Tongji University, Shanghai, China \\ Correspondence should be addressed to Ke Guan; kguan@bjtu.edu.cn
}

Received 25 June 2017; Revised 2 October 2017; Accepted 12 October 2017; Published 5 December 2017

Academic Editor: Ville Syrjälä

Copyright (C) 2017 César Briso-Rodríguez et al. This is an open access article distributed under the Creative Commons Attribution License, which permits unrestricted use, distribution, and reproduction in any medium, provided the original work is properly cited.

\begin{abstract}
Railway, subway, airplane, and other transportation systems have drawn an increasing interest on the use of wireless communications for critical and noncritical services to improve performance, reliability, and passengers experience. Smart transportation systems require the use of critical communications for operation and control, and wideband services can be provided using noncritical communications. High speed train (HST) is one of the best test cases for the analysis of communication links and specification of the general requirements for train control and supervision, passenger communications, and onboard and infrastructure wireless sensors. In this paper, we analyze in detail critical and noncritical networks mainly using the HST as a test case. First, the different types of links for smart rail transportation are described, specifying the main requirements of the transportation systems, communications, and their applications for different services. Then, we propose a network architecture and requirements of the communication technologies for critical and noncritical data. Finally, an analysis is made for the future technologies, including the fifth-generation $(5 \mathrm{G})$ communications, millimeter wave (mmWave), terahertz (THz), and satellites for critical and high-capacity communications in transportation.
\end{abstract}

\section{Introduction}

Wireless technologies have been widely developed in the last years and now are ready to meet the increasing demands of communication services for control, operation, and maintenance of smart transportation systems [1]. Existing radio technologies include Wi-Fi (IEEE 802.11xx), WiMAX (IEEE 802.11xx), Long Term Evolution (LTE), wireless sensor networks, wireless ad hoc networks, and particularly future fifth-generation (5G) technologies that will highly focus on the development of intelligent transportation systems of terrestrial and aerial vehicles. These emerging technologies can significantly improve the operation, efficiency, reliability, and passengers' experiences, but each communication network must be designed and configured to meet the special requirements of each transportation system.

Nowadays, transportation systems have an important demand of communications with very strict requirements of quality, capacity, and reliability. One of the best "test cases" for the analysis of communications in transportation systems is high speed train (HST). In a HST communication network, we can distinguish two types of communications: critical and noncritical communications.

Critical communications between HST and infrastructure are used for the control signaling [2] to increase speed, efficiency, safety, and reliability. These communications are critical because they are necessary for the high-performance operation of the transportation system so that it must have a very high reliability and availability (>99\%). Nevertheless, it is important to remark that communications are not responsible for the security. Security depends on other systems installed onboard or in the infrastructure. For example, if we consider a HST, Global System for Mobile Railway (GSMR) communications are used now to transmit telemetry (position) of the train and to send movement authorizations to it. This information is necessary to increase the speed of 
the train higher than $300 \mathrm{~km} / \mathrm{h}$ and must be updated every $100 \mathrm{~ms}$. If it is not updated during 1 second, there will be an emergency process reducing the speed of the train to a safe value [2]. To guarantee this performance it is necessary to achieve very high quality communications systems with redundancy and improved reliability. GSM-R is widely used in HST, but, currently, railway operators want to improve the performance of the trains and move to automatic driving so that they need a new high-capacity wireless communication system able to include high quality video transmissions from train to control center.

Another example could be the use of Unmanned Aerial Vehicles (UAVs) to improve security and reliability of smart rail transportation. As proposed in [3], these platforms can be used to enable and improve the communications and operation of next generation of smart transportation systems [3]. For these applications, UAVs need also a critical communication link and a payload link. Critical communication link is used to send position of the UAV from air to ground, and to send control commands or waypoints to the UAV [4]. There will be also a noncritical communication link that can be used for several purposes like video transmission for railway incident or infrastructure monitoring, or as a moving relay for emergency communication.

Thus, in both cases, the use of critical communications is mandatory, and these communications must have high quality of service because although security does not depend on them, frequent fails on communications can reduce reliability. For these reasons, a critical communication system has very special requirements and must rely on a very well proved technology specially customized for the smart transportation system. For example, HSTs are still using GSM-R standard, and commercial UAV radios use mainly early frequency hopping technology.

Noncritical communications are used for payload, supplementary services, and passenger services [5]. In this case, we require broadband communications for supplementary services like high-definition video and data services of the passengers. Some examples could be a video link transmitted by a UAV or a wideband Internet for the passengers of a high speed train. In both cases the principal requirement is capacity, so the design of these communications is completely different. Currently, these communications are on continuous development and are one of the principal challenges for the next years and for $5 \mathrm{G}$ technologies.

Finally, wireless sensors and ad hoc networks used for security and monitoring are now being used to provide supplementary services to vehicles $[5,6]$, and the use of millimeter wave (mmWave), terahertz (THz), and satellites will be relevant in the future to provide vehicle-to-vehicle communications, radar sensors, and onboard communications for different vehicle applications.

The remainder of this paper is organized as follows. Communications including critical and noncritical communications for smart rail transportation are comprehensively discussed in Section 2, in terms of their requirements, stateof-the-art development, trends, and challenges. Section 3 analyzes the requirements and principles of network planning for critical and noncritical communications. The principal techniques used and defined for network radio planning are highlighted as well. Conclusions are drawn in Section 4.

\section{Communications for Smart Rail Transportation}

As transportation systems become more automated, vehicles have an increasing demand on communications and are being equipped with more wireless communications services and sensors [7]. However, the maximum data rate of commercial communication systems (e.g., 4G) is still limited to $100 \mathrm{Mb} / \mathrm{s}$ for high mobility, and, therefore, current technologies are not sufficient to handle the terabyte-per-hour data rates that can be generated in next generation vehicles. Likewise, railway communications are required to provide various high data rate applications for passengers and train service. These applications need to be implemented in five rail scenarios (see Figure 1):

(i) Train-to-infrastructure (T2I) (HD video and other information in real time transmitting among various infrastructures)

(ii) Interwagon (wireless network between wagons)

(iii) Intrawagon (links between user equipment and access points of a wagon)

(iv) Inside station (links between access points (APs) and user equipment (UEs) in train/metro stations)

(v) Infrastructure-to-infrastructure (I2I) (HD video and other information in real time transmitting among various infrastructures) (note that whether this I2I scenario belongs to railway communication depends on whether this link is managed by railway industry; in most countries, the I2I link is provided by communication network operator, but in some countries, like China, such link is owned and managed by railway corporations.)

For the scenarios "inside station" and "infrastructure-toinfrastructure," the bandwidth requirements are from several hundred $\mathrm{MHz}$ to several $\mathrm{GHz}$, depending on concrete conditions. For the scenarios "intrawagon" and "interwagon," $1-10 \mathrm{GHz}$ bandwidths will be required, respectively. As the main interface between the network on train and the fixed network, the scenario "train-to-infrastructure" transmits an aggregated stream of the inter/intrawagon scenarios. Therefore, it requires the bandwidth of several $\mathrm{GHz}$ to accommodate over $100 \mathrm{Gbps}$ data rates [8]. Such high data rate and huge bandwidth requirements form a strong motivation of exploring the underutilized mmWave and even $\mathrm{THz}$ bands [8]. From the wireless channels viewpoint, there are numerous open challenges and chances on researching and developing mmWave and $\mathrm{THz}$ terrestrial and satellite communications enabling smart rail transportation [9]. Among all the above five scenarios, the most challenging one is the T2I scenario. As the main interface between the network on train and the fixed network, the T2I channel suffers strong dynamics, Doppler spread, and shadowing. Thus, it has drawn great attention, and, therefore, we mainly discuss it in the rest of the 

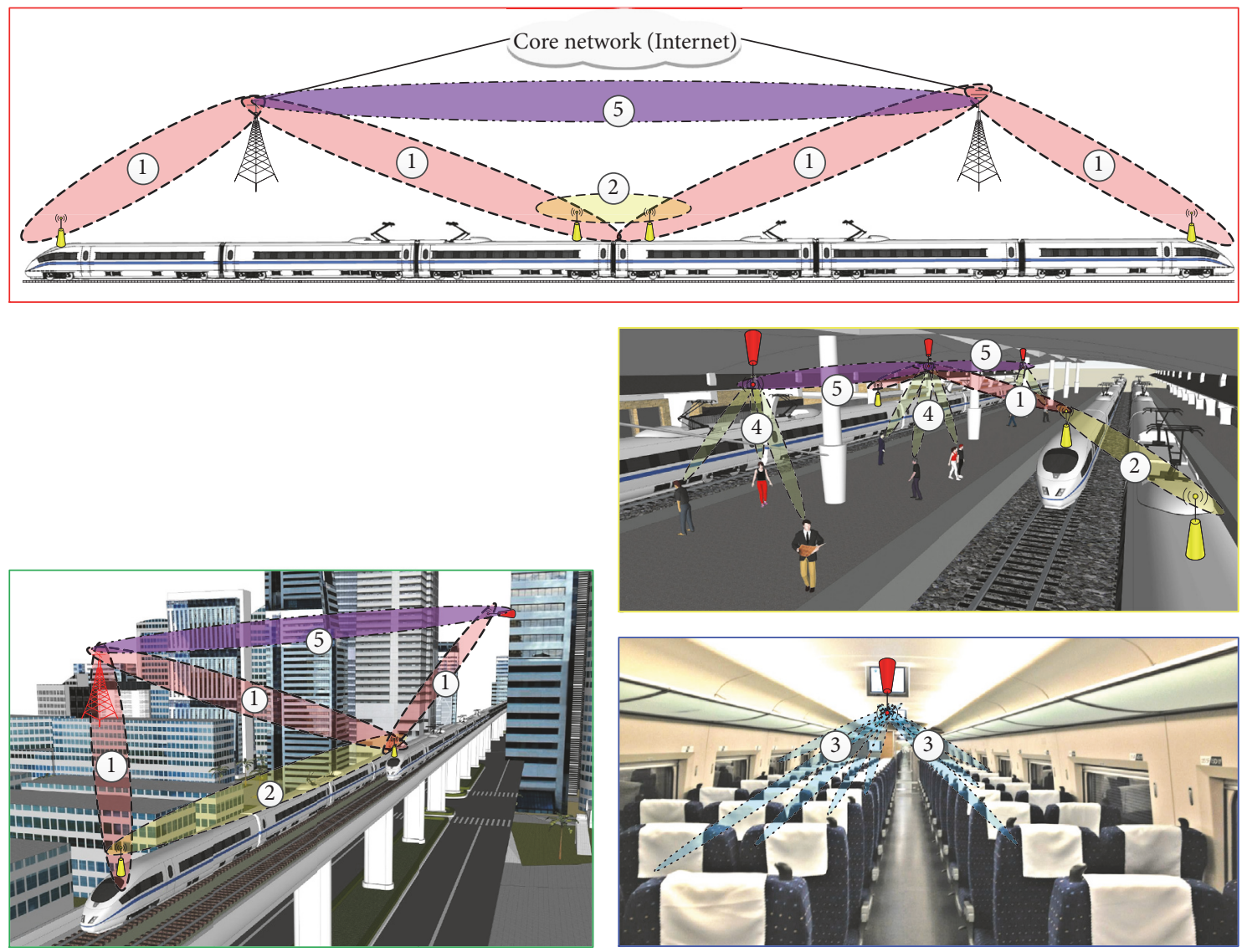

(1) Train-to-infrastructure

(2) Interwagon

(3) Intrawagon

(4) Inside station

(5) Infrastructure-to-infrastructure

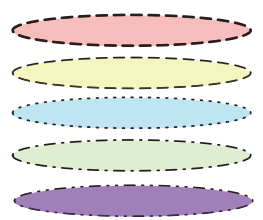

FIGURE 1: Five communication scenarios of smart rail transportation.

paper, rather than briefly talking about all of the five scenarios with equal weight.

2.1. Critical Communications. In contrast to the rapid and innovative changes in commercial mobile communications, the development of critical communications in public transportation has been rather more measured, mainly because of the mission-critical requirements. Among all the critical communications in railway, the GSM-R is the most widely used standard, and its stability has been verified for more than 10 years $[10,11]$. The principal specifications of a GSM$\mathrm{R}$ network for critical communications are summarized in Table 1 . However, due to limited transmission capacity, GSM$\mathrm{R}$ has been applied mainly to data communications for train control.

In order to provide new railway services such as video supervision and real-time monitoring, research efforts are now oriented towards LTE as the next generation technology [12-14]. In particular, the capabilities of LTE in terms of
TABLE 1: Quality of service performances of GSM-R system for critical communications.

\begin{tabular}{lc}
\hline GSM-R QoS parameter for ETCS & Requirement \\
\hline Connection establishment & $<8.5 \mathrm{~s}(95 \%)$ \\
& $\leq 10 \mathrm{~s}(100 \%)$ \\
\hline Transfer Delay (TD) & $\leq 0.5 \mathrm{~s}(99 \%)$ \\
\hline & $\leq 30 \mathrm{~s}(95 \%)$ \\
Network Registration Delay (NRD) & $\leq 35 \mathrm{~s}(99 \%)$ \\
& $\leq 40 \mathrm{~s}(100 \%)$ \\
\hline Connection Loss Rate (CLR) & $<10^{-2} / \mathrm{h}$ \\
\hline Transmission Interference (TI) & $<10^{-2}$ \\
\hline
\end{tabular}

future railway operational needs are currently being reviewed under the Future Railways Mobile Communications System (FRMCS) project, which was initiated by UIC in 2013. Recently, the authors in [15] have proposed LTE Railway (LTE-R) as an integrated wireless railway communications 
system by validating a deployed testbed of LTE-R. Moreover, "signal quality on the top of and inside train," "chain type network deployment," and "guaranteeing quality of services (QoS) for safety services" are the main critical issues that need further and deeper technical consideration:

(i) Signal quality on the top of and inside train: when using the direct access mode for both the onboard terminals (usually on the top of the train or car) and mobile terminals inside the train or car, the most critical issue during cell optimization is the achievement of a target level of received signal strength for both onboard and mobile terminals. This is mainly because the signal from the base station that penetrates directly into the car suffers the penetration loss up to $24 \mathrm{~dB}$ [12]. Such difference of the two channels, on the one hand, poses much higher requirements for the transmission power of the base station and, on the other hand, requires careful design of the antenna parameters of the two types of receivers in order to ensure a certain level of signal quality simultaneously for both onboard and mobile terminals. When using the two-hop access mode-one T2I hop and one intrawagon hop-such challenge coming from the huge penetration loss of the train body can be avoided.

(ii) Chain type network deployment: rather than the hexagonal type cell structure in commercial networks, railway (or vehicular) dedicated communication networks use a cell structure of a sequential chain type. Because of this uniqueness of a dedicated network, the communication scheme has the chance to be optimized further with low complexity and without loss of optimality [16].

(iii) Guaranteeing QoS for ultrareliable low latency applications: safety-critical vehicular applications can be broadly classified as vehicle detection, road detection, lane detection, pedestrian detection, drowsiness detection, collision avoidance, and so forth [17]. All these applications assist drivers and reduce potential accident risks and therefore require ultrareliability and low latency (e.g., the allowable latency for Traffic Signal Violation Warning (V2I) is $100 \mathrm{~ms}$, while the allowable latency for vehicle collision warning (V2V) is $20 \mathrm{~ms}$ ). For railway, typically all safety-based services need the highest safety level (SIL4 [18]), low bandwidth (less than $1 \mathrm{~kb} / \mathrm{s}$ per train), significant delay constraints (less than $500-800 \mathrm{~ms}$ in the worst case, usually even less), and the traffic pattern is usually real-time variable bit rate (RT-VBR). Voice calls need higher data rates (i.e., $64 \mathrm{~kb} / \mathrm{s}$ depending on the codec). A good reference for maximum jitter could be $30 \mathrm{~ms}$ [19]. Therefore, in order to support these services, it is important to keep data connectivity at the highest priority level. To do so, the network design for critical communications in public transportation must make use of sophisticated network management schemes for handling exceptional situations such as traffic congestion.
TABLE 2: Principal characteristics of HST and infrastructure.

\begin{tabular}{lc}
\hline \multicolumn{2}{c}{ Characteristics of HST } \\
\hline Number of passengers & 1500 \\
Passengers per car & 75 \\
Number of cars & 20 \\
Maximum length & $750 \mathrm{~m}$ \\
Maximum speed & $350 \mathrm{~km} / \mathrm{h}$ \\
\hline \multicolumn{2}{c}{ Characteristics of the track } \\
\hline Maximum slope & $6 \%$ \\
Tunnels & $10-30 \%$ \\
Typical curvature radius & $1 \mathrm{~km}$ \\
Viaducts & $10-30 \%$ \\
\hline
\end{tabular}

2.2. Noncritical, Broadband Communications. High-capacity communications networks for payload services and passengers on transportation are beginning to develop nowadays. Passengers on HSTs, metros, airplanes, and boats demand the use of communications like the ones they have in their private vehicles or at their homes. Also, transportation system operators demand supplementary services like the following:

(i) Onboard and wayside high-definition (HD) video surveillance that is critical for safety and security concerns (e.g., cars stuck on railway crossings, terrorist attacks).

(ii) Onboard real-time high data rate connectivity for web browsing, video conferencing, video broadcast, and so forth.

(iii) Real-time train dispatching $H D$ video between train and train control centers (TCCs) required for train dispatching and future driverless systems as well as journey information that are dynamically updated for all passengers.

However, the implementation of these communications in smart transportation systems has an important complexity because each transportation system has its own special characteristics: number of users, maximum speed, vehicle construction, vehicle infrastructure, and others. For these reasons, it is necessary to design and adapt the communication network for each specific transport system, and, therefore, we can use public networks for critical communications. As example of general requirements, we can use a modern HST whose main characteristics are summarized in Table 2.

According to the specifications of the transportation system, it is necessary to efficiently use the public terrestrial infrastructure combined with other technologies to increase capacity and performance. Therefore, the use of $4 \mathrm{G}$ LTE networks must be combined with moving relay solutions onboard trains [20] and then combined with satellite networks and other $5 \mathrm{G}$ terrestrial networks to improve capacity and to reduce cost. It is, therefore, necessary to provide broadband services to users considering the characteristics of this means of transport and its peculiarities, which for HST are summarized in a data capacity close to 1-10 Gbps to provide broadband services up to 1500 users, who are going 


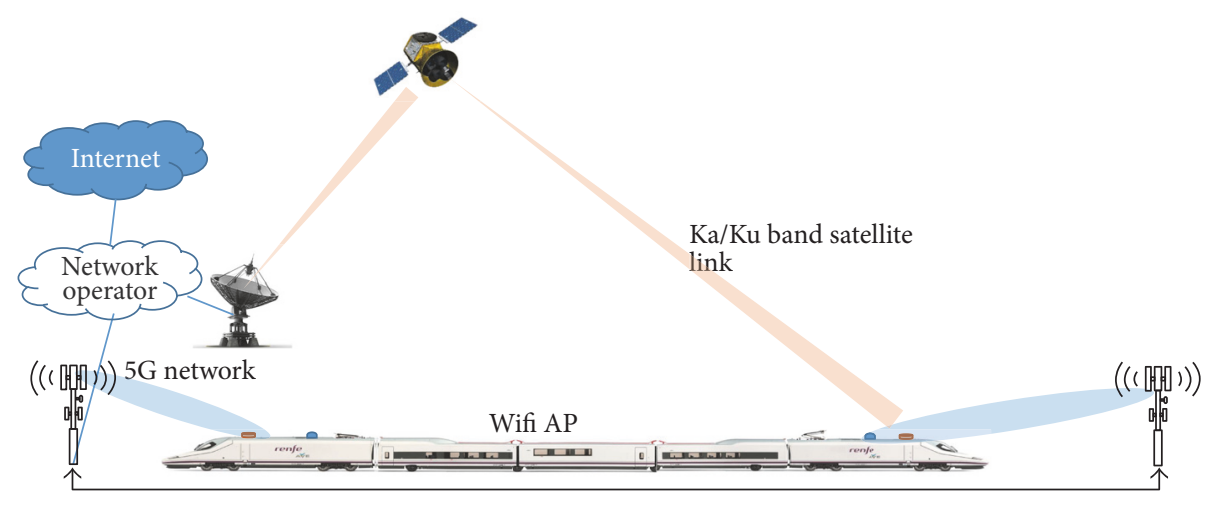

FIGURE 2: Broadband network for HST noncritical communications.

to maintain an intense connectivity. To this end, there is the problem of HST which makes it difficult to use terrestrial networks on desserts or inhabited areas that are frequently crossed by new HST. On the other hand, the frequent tunnels make it difficult to use the satellite, so a mixed solution must be used [21]. An example of architecture of such networks is shown in Figure 2.

This network must be capable of providing broadband communications to train passengers; for this purpose it will have a redundant terrestrial link that connects to the first and last wagon of the train. This link will use a moving relay solution in order to achieve reliable communications and reduce fixed infrastructure requirements. This network allows users to access a high-capacity terrestrial communications network that it is distributed inside the train using a wireless network based on the new high-capacity 802.11 standards such as 802.11ac/ad.

The satellite link is used as backup link for the terrestrial network. This link currently allows up to $100 \mathrm{Mbps}$ using antennas with automatic azimuth control and low profile elevation that can be simply installed on top of the train [22], and in the future, the capacity will be increased to 1 Gbps.

The entire communication system requires the use of an IP network along the train with a minimum capacity of 10 Gbps. This will allow managing the traffic of different links: train-land, train-satellite, and intratrain. Interwagon communications can be also done using short distance $10 \mathrm{Gbps}$ wireless links at mmWave or $\mathrm{THz}$, to allow train assembly and to minimize wired connections on trains wagons. Table 3 summarizes the requirements of a communication network for noncritical communications in HST

Trains will have an internal $10 \mathrm{Gpbs}$ network for multimedia applications of the passengers, with an external connection of $1 \mathrm{Gbps}$ for Internet connections. Also, in the station, there will be a 100 Gbps network for all the train noncritical services. This station network can have many infrastructureto-infrastructure communications for additional services as passengers transfer, video surveillance, advertising screens, digital TV, and other new services.

As an example of terrestrial network, we can describe the current LTE network that can reach capacities of $100-300 \mathrm{Mbps}$ using carrier aggregation on the $1-6 \mathrm{GHz}$
TABLE 3: Requirements of a communication network for noncritical communications in HST.

\begin{tabular}{lc}
\hline \multicolumn{2}{c}{ HST Communication Links } \\
\hline Train-to-infrastructure (terrestrial) & $1 \mathrm{Gbps}$ \\
Train-to-infrastructure (satellite) & $0.2 \mathrm{Gbps}$ \\
Intra wagon & $1 \mathrm{Gbps}$ \\
Inter wagon & $10 \mathrm{Gbps}$ \\
Inside station (all commuters) & $100 \mathrm{Gbps}$ \\
Infrastructure-to-infrastructure & $10 \mathrm{Gbps}$ \\
\hline
\end{tabular}

band. This performance can be efficiently used on HST using a moving relay solution on board the train. These links allow reliable communications up to speeds of $350 \mathrm{~km} / \mathrm{h}$ with the problem that throughput is reduced with velocity when the channel is degraded, as described in [23]. To get higher throughputs, it is necessary to use lower frequency and higher frequency networks together. In Table 4, we include a comparison of the key parameters of four radio technologies, two suitable for critical communications and two for noncritical communications. As we can see in this table, the critical network has been configured for high quality of service, low delay, and high reliability, while the noncritical networks are developed for high capacity and, therefore, have a high demand of bandwidth. For this reason, noncritical communications are considering the use of mmWave technologies in the $28-34 \mathrm{GHz}$ band because it is suitable for mobile communications.

The use of $\mathrm{mmW}$ for mobile communications in high mobility environments requires the use of high gain antennas with phased array technology, and this technology is very suitable for communications of high capacity in environments like those of the HST where the coverage is linear. It will allow the availability of high bandwidth, getting data rates on the Gbps range [24]. In summary, broadband communications in a transport system such as the HST require a complex project where it is necessary to install a network inside the train and several external networks combining terrestrial links in microwave and mmWave bands together with satellite links. 
TABLE 4: Example of different wireless communications systems customized for critical and noncritical communications.

\begin{tabular}{|c|c|c|c|c|c|}
\hline \multirow{2}{*}{ Parameter } & \multicolumn{2}{|c|}{ Critical } & \multicolumn{3}{|c|}{ Noncritical } \\
\hline & GSM-R & LTE-R & LTE & WLAN 802.11xx & Satellite \\
\hline Frequency & $\begin{array}{l}\text { Uplink: } 876-880 \mathrm{MHz} \\
\text { downlink: } 921-925 \mathrm{MHz}\end{array}$ & $\begin{array}{c}450 \mathrm{MHz}, 800 \mathrm{MHz}, \\
1.4-1.8 \mathrm{GHz}\end{array}$ & $\begin{array}{c}800 \mathrm{MHz}, 1.8 \mathrm{GHz}, \\
2.6 \mathrm{GHz}\end{array}$ & $\begin{array}{c}\text { 2.4/5.7 GHz, } \\
24 / 28 / 33 \mathrm{mmW}\end{array}$ & $12 / 14 \mathrm{GHz}$ \\
\hline Bandwidth & $0.2 \mathrm{MHz}$ & $1.4-20 \mathrm{MHz}$ & $1.4-20(100) \mathrm{MHz}$ & $80(160) \mathrm{MHz}$ & $10-16 \mathrm{MHz}$ \\
\hline Modulation/multiple & GMSK & QPSK & M-QAM & M-QAM & QPSK \\
\hline access & $\mathrm{FDD}+\mathrm{TDM}$ & $\mathrm{FDD}+\mathrm{OFDM}$ & $\mathrm{FDD}+\mathrm{OFDM}$ & TDD + OFDM & FDD \\
\hline $\begin{array}{l}\text { Peak data rate, } \\
\text { downlink/uplink }\end{array}$ & 172/172 Kbps & 50/10 Mbps & 100/50 Mbps & 433.3 Mbps & 10/1 Mbps \\
\hline $\begin{array}{l}\text { Peak spectral } \\
\text { efficiency }\end{array}$ & $0.33 \mathrm{bps} / \mathrm{Hz}$ & $2.55 \mathrm{bps} / \mathrm{Hz}$ & $16.32 \mathrm{bps} / \mathrm{Hz}$ & $4.8 \mathrm{bps} / \mathrm{Hz}$ & $1 \mathrm{bps} / \mathrm{Hz}$ \\
\hline $\begin{array}{l}\text { Maximum } \\
\text { transmission delay }\end{array}$ & $<50 \mathrm{~ms}$ & $<100 \mathrm{~ms}$ & $<1 \mathrm{~s}$ & $<1 \mathrm{~s}$ & $<1.5 \mathrm{~s}$ \\
\hline Data transmission & $\begin{array}{l}\text { Requires voice call } \\
\text { connection }\end{array}$ & Packet switching & Packet switching & Packet switching & Packet switching \\
\hline Packet retransmission & No (serial data) & $\begin{array}{l}\text { UDP packets, } 0 \\
\text { retransmissions }\end{array}$ & $\begin{array}{l}\text { IP packets with up } \\
\text { to } 5 \text { retransmissions }\end{array}$ & $\begin{array}{l}\text { IP packets, up to } \\
5 \text { retransmissions }\end{array}$ & $\begin{array}{l}\text { IP packets, } 0 \\
\text { retransmissions }\end{array}$ \\
\hline MIMO & $\begin{array}{c}\text { SISO } \\
\text { (diversity) }\end{array}$ & $2 \times 2$ & $2 \times 2,4 \times 2$ & $4 \times 4$, up to 15 & no \\
\hline Mobility & Max. $500 \mathrm{~km} / \mathrm{h}$ & Max. $500 \mathrm{~km} / \mathrm{h}$ & Max. $350 \mathrm{~km} / \mathrm{h}$ & Max. $250 \mathrm{~km} / \mathrm{h}$ & $>500 \mathrm{Km} / \mathrm{h}$ \\
\hline Handover success rate & $\geq 99.5 \%$ & $\geq 99.9 \%$ & $\geq 99.5 \%$ & $\geq 99.5 \%$ & - \\
\hline Handover procedure & Hard & Soft & Hard/soft & Hard/soft & No handover \\
\hline All IP (native) & No & Yes & Yes & Yes & yes \\
\hline
\end{tabular}

\section{Network Design for Critical and Noncritical Communications}

The design of critical and noncritical communications is completely different from conventional networks. In the first case, it is necessary to make a special design of the network focused on QoS and reliability. In the other case, noncritical communications are focused on capacity. In both cases, the model and design of the communication network are completely different, considering node architecture, handover schemas, relay configuration, Multiple-Input MultipleOutput (MIMO), antennas, and diversity. Then, it is necessary to accurately design the physical interface for each special environment, vehicle dynamic, and passengers or payload requirements. It includes propagation modeling, waveform selection, and antenna design for current communications bands $(1-6 \mathrm{GHz})$, mainly for critical communications and for future mmWave bands $(24-34 \mathrm{GHz})$ for passengers and payload $[25,26]$.

High quality of service networks is based on the use of redundant communications to increase reliability and maintainability. For this purpose, railway critical communications use two equal terrestrial networks simultaneously. This solution guarantees an excellent radio coverage and reliability.

Noncritical communications can also use two or more networks at the same time, but, in this case, the design of the network is focused to increase capacity, so two or more networks work in parallel and at the same time guarantee a minimum coverage in complex areas. Usually, high-capacity

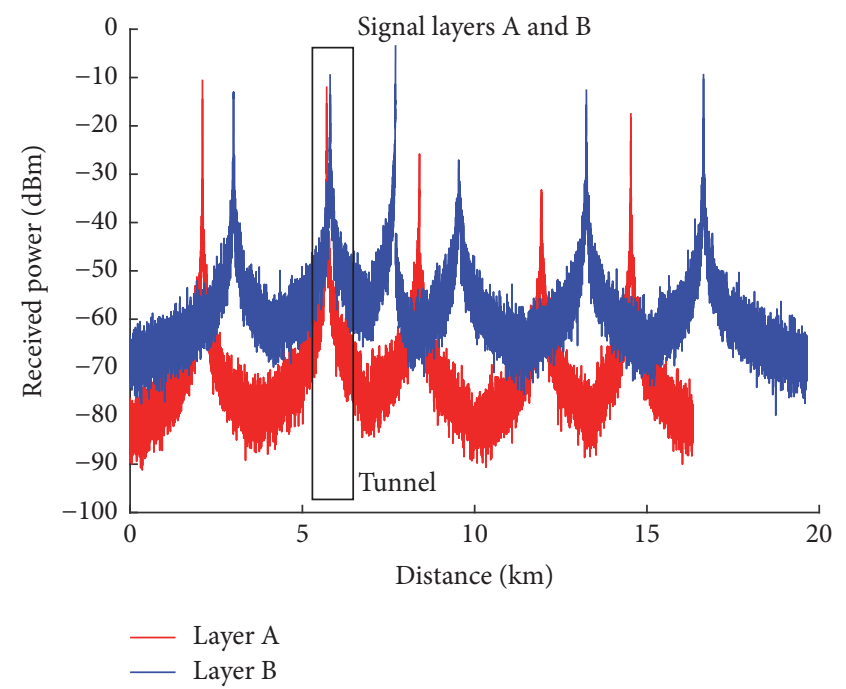

FIGURE 3: Dual layer network measurements for HST critical communications.

terrestrial network can be complemented with a satellite link to provide radio coverage on remote areas and with other terrestrial networks to improve capacity on special parts of the track like stations or densely populated areas.

Figure 3 shows the measurements of a dual layer network for HST GSM-R communications [27]. We can see that the two layers are quite well interlaced in the entire track except on the tunnel where there is a double repeater on 


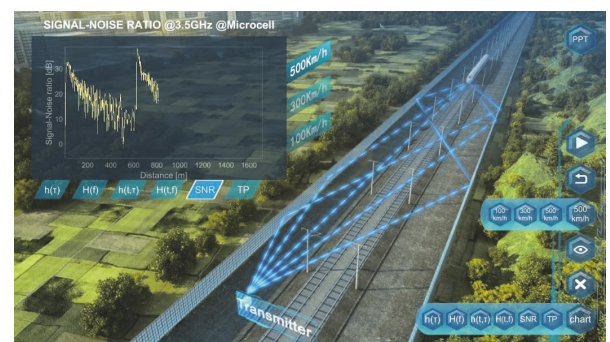

Figure 4: An example of realizing network planning of HST communications based on ray-tracing channels.

the middle of the tunnel. With this design, the train is continuously connected to both networks, increasing the QoS and availability to $98 \%$, compared with $86 \%$ for a single network. In case of fail of a base station or a complete layer, the other can support the communications without degradation of critical communications.

The design of noncritical communications will require a different solution. In this case, the two networks will be overlapped with the same radio coverage to maximize capacity of the users instead of QoS and reliability. For both wireless networks used in critical or noncritical communications, it is necessary to make a careful network planning using deterministic channel modeling approaches. New techniques of radio planning, such as ray-tracing and algorithms for railway propagation as proposed in [29], may act as the first choice for realizing accurate link budget. Figure 4 illustrates an example of realizing network planning of HST communications based on ray-tracing channels. The principal techniques used and defined for network radio planning can be summarized as follows:

(i) To include the road/railway features in channel models: this is an open challenge mainly caused by the lack of standard channel models and reference scenarios exclusively for roads/railways. Beijing Jiaotong University has defined and constructed a set of reference scenario modules for mmWave and $\mathrm{THz}$ terrestrial and satellite railway channels [30]. These modules include all the main objects, such as tracks, stations, crossing bridges, tunnels, cuttings, barriers, pylons, buildings, vegetation, traffic signs, billboards, trains, etc., with their typical geometries and materials in reality. They can either be independently used for site-specific verification or be combined in various ways for doing statistical analysis. Some snapshots of ray-tracing simulations in these HST scenarios are shown in Figure 5. The 3D environment models are publicly available and freely downloadable with the link http://raytracer.cloud.

(ii) Design of mmWave and $\mathrm{THz}$ fixed and mobile networks: these frequencies require an accurate and efficient radio planning, and ray-tracing simulations are very useful at this point but accurate simulations are very challenging, because a large number of objects will influence channel properties, and, therefore, they should be included in the scenarios $[31,32]$. In order to tackle this challenge, to evolve the deployment of the RT simulator from on PC to on high-performance computing (HPC) platform is a promising solution. The authors of [9] present the state-of-the-art development of the high-performance RT platform. In the case study of doing RT simulations in the three railway scenarios at $30 \mathrm{GHz}$, the high-performance RT platform is 100 times faster than the laptop-based RT and without "out of memory" issue.

(iii) In the current road/railway network deployment, very fast adaptive beamforming will be needed due to the high mobility of the car/train. However, there is a chance of avoiding the requirement of such challenging beamforming, which is deploying the transmitting $(\mathrm{Tx})$ antennas towards the same direction along the road/rail and moving the handover from the middle of two base stations (BSs) to the region when the train is very near the BS. With this innovation, both RT simulations (see Figure 6 which is Figure 9 in [9]) and measurements which are done in Seoul subway line 8 by Electronics and Telecommunications Research Institute (ETRI) (see Figure 7 which is Figure 9 in [28]) imply that even the fixed directional antenna (or beamforming) can support the link length longer than $1 \mathrm{~km}$ under lineof-sight (LOS) condition and around several hundred meters under non-LOS (NLOS) condition at $30 \mathrm{GHz}$.

(iv) Compared with the high cost of deploying dedicated mmWave and $\mathrm{THz}$ terrestrial communications, satellites are a good solution because of no need for a terrestrial network (even though a backup technology is needed when cars/trains are in tunnels). Many research projects have explored the possibility of providing Internet access to onboard passengers [33], but most of them have failed, due to either the technical complexity or the lack of an adequate business plan [33]. Recently a new telecommunication solution based on multiple radio bearers using cellular and satellite public networks as an alternative to the deployment of a dedicated infrastructure is proposed in [21]. A test campaign on $300 \mathrm{~km}$ of rail line (about $10,000 \mathrm{~km}$ of tests) has been carried out to evaluate the performance of cellular and satellite networks in a railway environment. Results in terms of achievable link reliability and end-to-end (E2E) delays are encouraging for the adoption of a public land mobile network (PLMN)/satellite approach to export ERTMS/ETCS procedures [22].

\section{Conclusions}

In this paper, the state-of-the-art wireless communications in smart rail transportation systems are surveyed and discussed comprehensively. To begin with, the communication requirements and scenarios/links of smart rail transportation are defined in terms of train-to-infrastructure, interwagon, intrawagon, inside station, and infrastructureto-infrastructure scenarios. Then, regarding the critical 

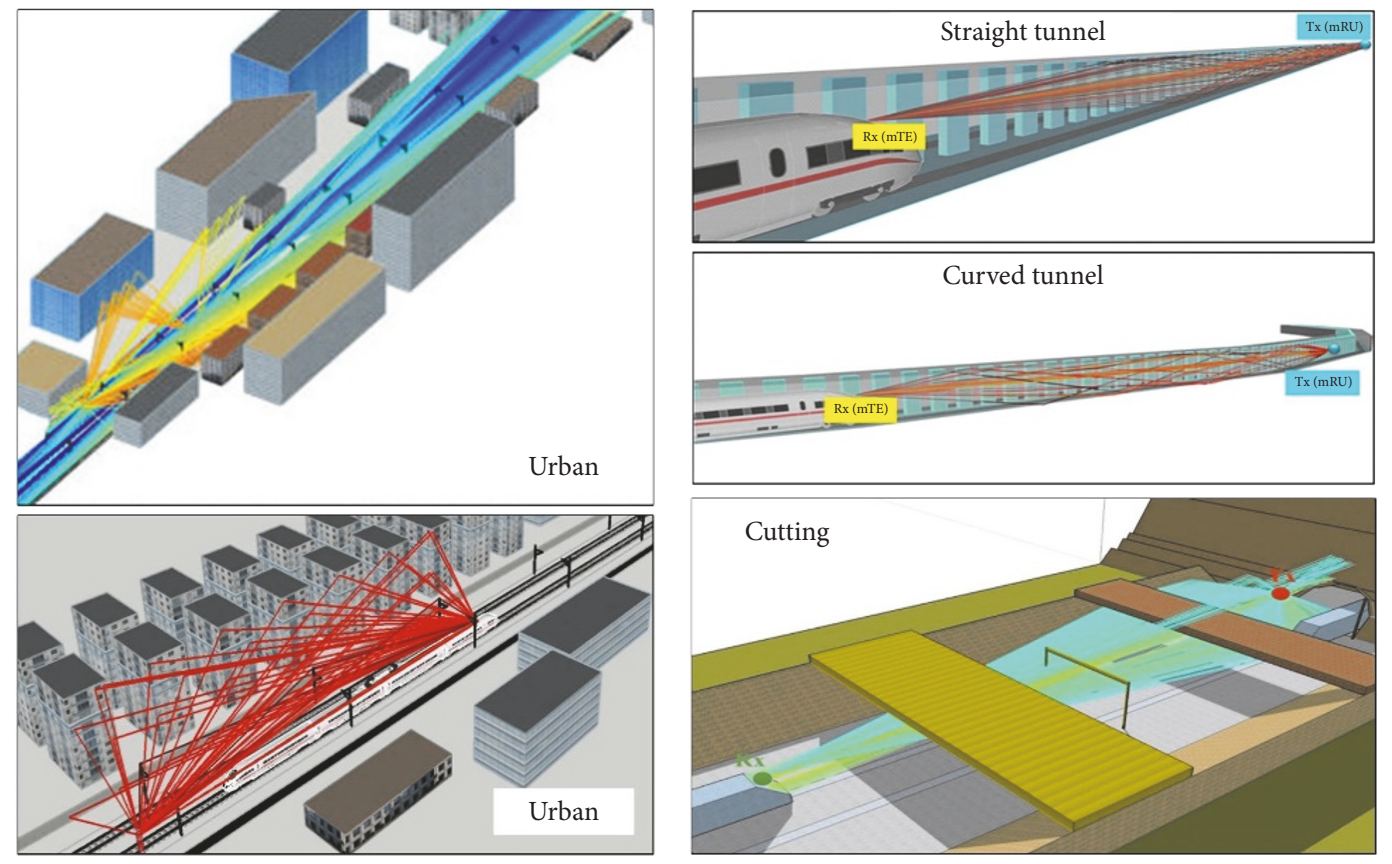

FIGURE 5: Snapshots of ray-tracing simulations in some HST scenarios.

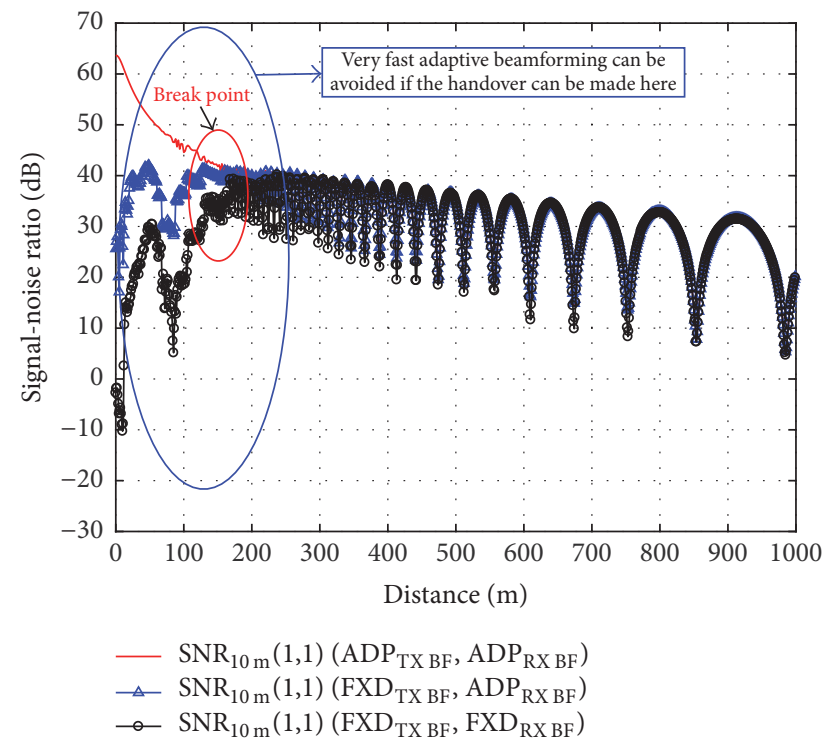

FIGURE 6: Signal-noise ratio in the railway urban scenario at $30 \mathrm{GHz}$ using different beamforming strategies [9].

communications, the situation of current GSM-R and the trend of future LTE-R are reviewed, and the main critical issues-signal quality on the top of and inside train, chain type network deployment, and guaranteeing QoS for safety services-are highlighted for further study. Moreover, multiple potential solutions of providing noncritical, broadband communications are analyzed. A complex project is highly required to install a network inside the train and several external networks combining terrestrial links in microwave and mmWave bands together with satellite links. For the

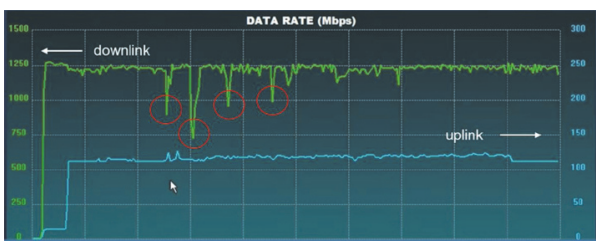

Figure 7: Measured data throughput during $2.4 \mathrm{~km}$ run [28].

network planning, usage of redundant communications is the key solution of guaranteeing an excellent radio coverage and reliability of critical communications, while deploying two or more different networks that can work in parallel is of importance of providing noncritical, broadband communications to the onboard users. Last but not least, some principal techniques are highlighted for network radio planning for smart rail transportation systems, including accurate link budget using high-performance ray-tracing methods, new handover scheme avoiding very fast adaptive beamforming and new telecommunication solutions based on multiple radio bearers using cellular and satellite public networks.

\section{Conflicts of Interest}

The authors declare that they have no conflicts of interest.

\section{Acknowledgments}

This work was developed under the framework of ENABLING 5G TEC2014-55735-C3-2-R funded by the Spanish Ministry of Economy and Competitiveness and by NSFC under Grants 61771036 and 61501021, Beijing Natural 
Science Foundation under Grant L161009, Alexander von Humboldt Foundation, and State Key Lab of Rail Traffic Control and Safety Project under Grants RCS2017ZZ004 and RCS2017ZT008.

\section{References}

[1] E. Aguirre, P. Lopez-Iturri, L. Azpilicueta et al., "Design and implementation of context aware applications with wireless sensor network support in urban train transportation environments," IEEE Sensors Journal, vol. 17, no. 1, pp. 169-178, 2017.

[2] A. D. Zayas, C. A. G. Pérez, and P. M. Gómez, "Third-generation partnership project standards: for delivery of critical communications for railways," IEEE Vehicular Technology Magazine, vol. 9, no. 2, pp. 58-68, 2014.

[3] H. Menouar, I. Guvenc, K. Akkaya, A. S. Uluagac, A. Kadri, and A. Tuncer, "UAV-Enabled Intelligent Transportation Systems for the Smart City: Applications and Challenges," IEEE Communications Magazine, vol. 55, no. 3, pp. 22-28, 2017.

[4] T. C. Hong, K. Kang, K. Lim, and J. Y. Ahn, "Network architecture for control and non-payload communication of UAV," in Proceedings of the 2016 International Conference on Information and Communication Technology Convergence (ICTC '16), pp. 762-764, IEEE, Jeju, South Korea, October 2016.

[5] L. Zhu, F. R. Yu, B. Ning, and T. Tang, "Communication-based train control (CBTC) systems with cooperative relaying: Design and performance analysis," IEEE Transactions on Vehicular Technology, vol. 63, no. 5, pp. 2162-2172, 2014.

[6] R. He, B. Ai, G. Wang et al., "High-Speed railway communications: from GSM-R to LTE-R," IEEE Vehicular Technology Magazine, vol. 11, no. 3, pp. 49-58, 2016.

[7] J. Choi, V. Va, N. González-Prelcic, R. Daniels, C. R. Bhat, and R. W. Heath, "Millimeter-wave vehicular communication to support massive automotive sensing," IEEE Communications Magazine, vol. 54, no. 12, pp. 160-167, 2016.

[8] K. Guan, G. Li, T. Kürner et al., "On millimeter wave and THz mobile radio channel for smart rail mobility," IEEE Transactions on Vehicular Technology, Early Access, 2017.

[9] K. Guan, D. P. He, A. Hrovat, B. Ai, Z. D. Zhong, and T. Kurner, "Challenges and chances for smart rail mobility at mmWave and $\mathrm{THz}$ bands from the channels viewpoint," in Proceedings of the 2017 15th International Conference on ITS Telecommunications (ITST '17), pp. 1-5, Warsaw, Poland, May 2017.

[10] ERTMS SUBSET-093 v2.3.0, GSM-R Interfaces: Class 1 Requirements, 2005.

[11] UIC CODE 950 v7.3.0, EIRENE Functional Requirements Specification, 2012.

[12] B. Ai, K. Guan, M. Rupp et al., "Future railway services-oriented mobile communications network," IEEE Communications Magazine, vol. 53, no. 10, pp. 78-85, 2015.

[13] B. Ai, X. Cheng, T. Kurner et al., "Challenges toward wireless communications for high-speed railway," IEEE Transactions on Intelligent Transportation Systems, vol. 15, no. 5, pp. 2143-2158, 2014.

[14] K. Guan, Z. Zhong, and B. Ai, "Assessment of LTE-R using high speed railway channel model," in Proceedings of the 2011 3rd International Conference on Communications and Mobile Computing (CMC '11), pp. 461-464, China, April 2011.

[15] Y.-S. Song, J. Kim, S. W. Choi, and Y.-K. Kim, "Long term evolution for wireless railway communications: testbed deployment and performance evaluation," IEEE Communications Magazine, vol. 54, no. 2, pp. 138-145, 2016.

[16] H. Maleki and S. A. Jafar, "Optimality of orthogonal access for one-dimensional convex cellular networks," IEEE Communications Letters, vol. 17, no. 9, pp. 1770-1773, 2013.

[17] C. Bila, F. Sivrikaya, M. A. Khan, and S. Albayrak, "Vehicles of the future: a survey of research on safety issues," IEEE Transactions on Intelligent Transportation Systems, vol. PP, no. 99, 2016.

[18] IEC 61508: 2010, Functional Safety of Electrical/Electronic/Programmable Electronic Safety-Related Systems, (parts 1-7), 2010.

[19] J. Moreno, J. Riera, L. De Haro, and C. Rodriguez, "A survey on future railway radio communications services: challenges and opportunities," IEEE Communications Magazine, vol. 53, no. 10, pp. 62-68, 2015.

[20] Y. Sui, J. Vihriala, A. Papadogiannis, M. Sternad, W. Yang, and T. Svensson, "Moving cells: a promising solution to boost performance for vehicular users," IEEE Communications Magazine, vol. 51, no. 6, pp. 62-68, 2013.

[21] F. Mazzenga, R. Giuliano, A. Neri, and F. Rispoli, "Integrated public mobile radio networks/satellite for future railway communications," IEEE Wireless Communications Magazine, vol. 24, no. 2, pp. 90-97, 2017.

[22] E. L. Cid, M. G. Sanchez, and A. V. Alejos, "Wideband analysis of the satellite communication channel at ku- and x-bands," IEEE Transactions on Vehicular Technology, vol. 65, no. 4, pp. 2787-2790, 2016.

[23] J. Rodríguez-Piñeiro, J. A. García-Naya, P. Suárez-Casal, C. Briso-Rodríguez, J. I. Alonso-Montes, and L. Castedo, "Assessment of channel propagation conditions for FDD LTE transmissions in the Spanish high-speed railways," in Proceedings of the 10th European Conference on Antennas and Propagation (EuCAP '16), pp. 1-5, IEEE, Davos, Switzerland, April 2016.

[24] J. Zhang, M. Xu, J. Wang et al., "Carrier aggregation for MMW inter-RAT and intra-RAT in next generation heterogeneous mobile data network based on optical domain band mapping," in Proceedings of the 2015 European Conference on Optical Communication (ECOC '15), pp. 1-3, Valencia, Spain, October 2015.

[25] P. T. Dat, A. Kanno, N. Yamamoto, and T. Kawanishi, "WDM RoF-MMW and linearly located distributed antenna system for future high-speed railway communications," IEEE Communications Magazine, vol. 53, no. 10, pp. 86-94, 2015.

[26] J. Zhang, P. Tang, L. Tian, Z. Hu, T. Wang, and H. Wang, " $6-100 \mathrm{GHz}$ research progress and challenges for fifth generation $(5 \mathrm{G})$ and future wireless communication from channel perspective," Science China Information Sciences, vol. 60, no. 8, pp. 1-16, 2017.

[27] A. Aziz, S. Rizvi, N. M. Saad, and B. B. Samir, "An overview of integrated architectures solutions in wireless heterogeneous networks," in Proceedings of the 2011 National Postgraduate Conference (NPC '11), pp. 1-4, IEEE, Kuala Lumpur, Malaysia, September 2011.

[28] H. Chung, J. Kim, G. Noh et al., "From architecture to field trial: A millimeter wave based MHN system for HST Communications toward 5G," in Proceedings of the 2017 European Conference on Networks and Communications (EuCNC '17), pp. 1-5, Oulu, Finland, June 2017.

[29] K. Guan, Z. Zhong, B. Ai, and T. Kürner, "Deterministic propagation modeling for the realistic high-speed railway environment," in Proceedings of the IEEE 77th Vehicular Technology Conference (VTC '13), pp. 1-5, Dresden, Germany, June 2013. 
[30] K. Guan, X. Lin, D. He et al., "Scenario modules and raytracing simulations of millimeter wave and terahertz channels for smart rail mobility," in Proceedings of the 2017 11th European Conference on Antennas and Propagation (EUCAP '17), pp. 113117, IEEE, Paris, France, March 2017.

[31] W. Fan, I. Carton, P. Kyösti, and G. F. Pedersen, "Emulating ray-tracing channels in multiprobe anechoic chamber setups for virtual drive testing," IEEE Transactions on Antennas and Propagation, vol. 64, no. 2, pp. 730-739, 2016.

[32] X. Chen and S. Zhang, "Multiplexing efficiency for MIMO antenna-channel impairment characterization in realistic multipath environments," IET Microwaves, Antennas \& Propagation, vol. 11, no. 4, pp. 524-528, 2017.

[33] D. T. Fokum and V. S. Frost, "A survey on methods for broadband internet access on trains," IEEE Communications Surveys \& Tutorials, vol. 12, 2nd qtr., no. 2, pp. 171-185, 2010. 


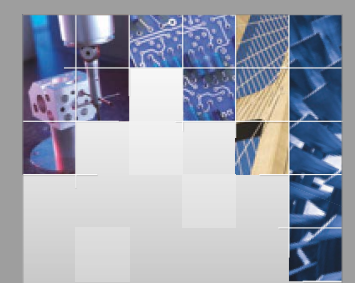

\section{Enfincering}
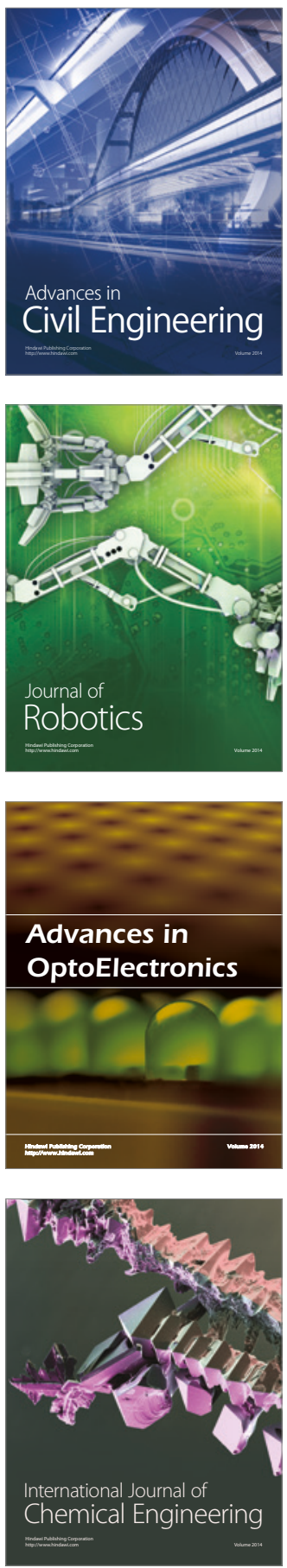

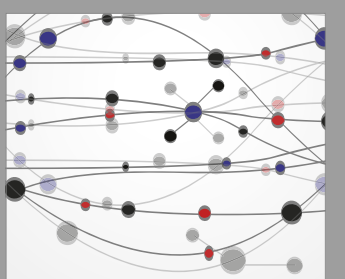

The Scientific World Journal

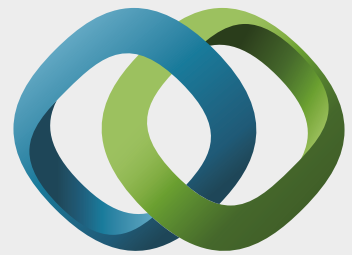

\section{Hindawi}

Submit your manuscripts at

https://www.hindawi.com
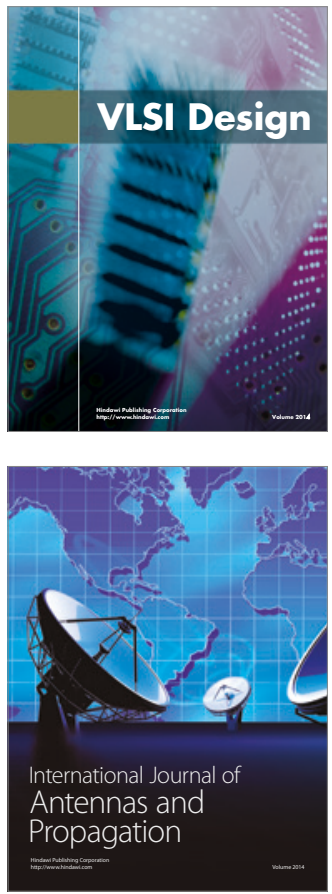

\section{Rotating}

Machinery
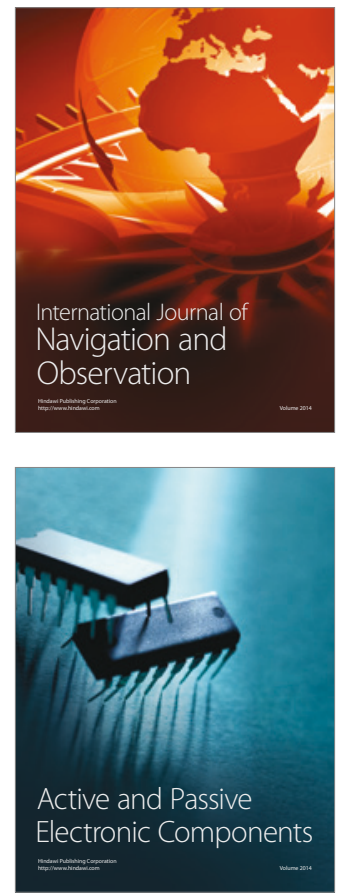
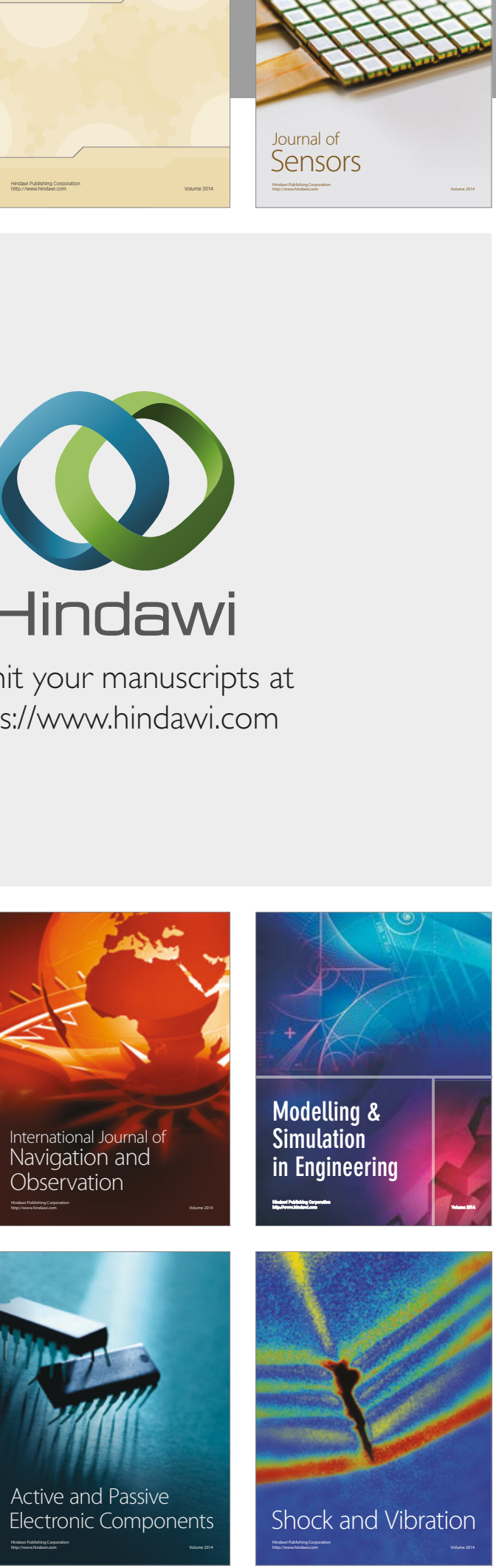
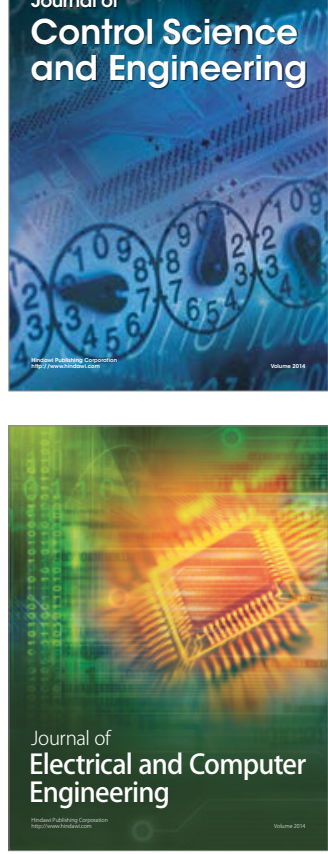

Distributed

Journal of

Control Science

and Engineering
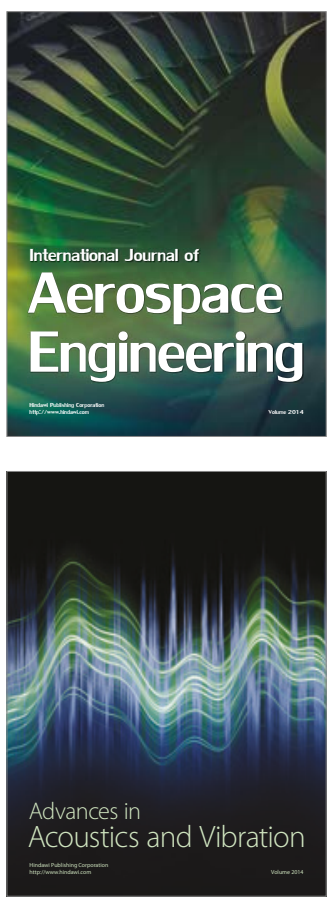

Sensor Networks 